\title{
Clinical Trial Initiation Monitoring Report
}

National Cancer Institute

\section{Source}

National Cancer Institute. Clinical Trial Initiation Monitoring Report. NCI Thesaurus. Code C115576.

Records pertaining to site initiation-related visits, and a site's completion of requirements to begin participation in a clinical trial, including confirmation that trial procedures have been reviewed by the investigator and trial personnel at the site. 